\section{Clinicians and The Modern EHR: Statistician, Scribe or Storyteller?}

Luay Shayya MD1, Shaheen E. Lakhan MD, PhD, MEd, MS*1,2, Deborah Tepper MD ${ }^{1}$

\title{
Abstract
}

The Health Information Technology for Economic and Clinical Health (HITECH) Act spearheaded the rapid adoption of electronic health records (EHR) in the United States. Although HITECH's mission is to implement data storage, order management, evidence-based decision support, electronic communication and connectivity, and administrative processes and reporting (i.e. billing and coding), the main purpose of the EHR for the practicing clinicians should remain the same -- to convey the story, facts, and plan for the patient's health care to the reader. We define three categories of EHR notes: the statistical, the scribe, and the storyteller styles, the pros and cons of each, and how the strengths of each may be used for more effective written communication.

\section{Introduction}

In 2009, the American Recovery and Reinvest Act (ARRA) was passed which included the Health Information Technology for Economic and Clinical Health (HITECH) Act. The HITECH Act led to the rapid and widespread adoption of EHR. Additional definitions then focused on the nature of the health record. This included targeted core capabilities such as health information and data storage, test management, order management, evidence-based decision support, electronic communication and connectivity, patient support, administrative processes and reporting (i.e. billing and coding), and public health management[1]. However, arguably the most important and apparent purpose of the health record for the practicing clinician should remain the same -- to convey the story, facts, and plan for the patient's health care to the reader. In an effort to balance these clinical needs with new core capabilities, we have been subject to an unwinnable written conflict between the need to communicate

1 Neurological Institute, Cleveland Clinic, Cleveland $\mathrm{OH}$.

2 Global Neuroscience Initiative Foundation, Los Angeles, CA.

Contact information:

Neurological Institute, Cleveland Clinic, Cleveland $\mathrm{OH}$.

Tel: (216) 444-2200.

Address: 9500 Euclid Ave, S100C, Cleveland $\mathrm{OH} 44195$.

झ slakhan@gnif.org 
large amounts of objective but often extraneous data, and providing a physician-biased filter that sorts out the pertinent and provides the reasoning for arriving at a diagnosis and plan. In this article we will outline three broad writing styles which capture this ongoing conflict. We hope that by demonstrating these pros and cons, modern clinicians will maximize the impact of their most valuable asset: their opinion. By looking at three categories of EHR notes, the statistical, the scribe, and the storyteller styles, we will see the pros and cons of each, and perhaps see how the strengths of each may be used for more effective written communication.

\section{The Statistician}

The statistical EHR note is a regimented approach, often characterized by checkboxes or lists. This can assist the writer and the readers ascertain the probability of diseases based upon the chosen symptoms. Some of the available EHRs will convert the checklists into pre-drafted sentences[2]. For example, if a patient presents with a headache, the note can be constructed to read: "New onset headache for 7 hours. History of migraines. Not similar to prior headaches. Frequency is constant. Progression since onset is rapidly worsening. Not associated with light/sound sensitivity, neck pain, weakness, numbness, myalgias, or paresthesias. Pain location is temporal. Pain quality is sharp. Numeric pain scale is $10 / 10$. Pain severity is severe. Pain does not radiate. Treatments tried include acetaminophen." The statistical note is very simplified, which assists with billing and coding. In addition, it can cover a broad range of differential diseases by offering the physician symptoms to consider. Unfortunately it risks being "devoid of any medical thought"[3]. The pre-drafted sentences are further proof of the dichotomous thought process seen in statistical writing. Using objective data, the statistical style tends to pull all data into the note. This lack of discrimination is one of the frequently cited flaws of an EHR, which is it does not sort out the important data from the less so. It is said to "...pull in so much information with the click of a button that the final note becomes an essentially unreadable tome through which one must search high and wide to find essential information"[4]. Pertinent data is intermixed with non-pertinent data; it is the readers' job to analyze and differentiate between the two data groups.

\section{The Scribe}

The second broad type of EHR note is the scribe's note. This takes into account that presenting the full range of data seen in the statistical note, as well as the time-consuming narrative descriptors in a story teller's note, can be excessive, impractical, and inefficient. Time limitations make recording raw data a priority, leaving the assumed data analysis to the reader's interpretation. Just as a scribe writes down what they hear verbatim, so does the hurried physician. Our patient with a headache may read as "Patient reports the headache is new. He has migraines, but this headache is different. Pt states the headache is like an ice-pick, doesn't move, is constant and has progressively gotten worse for the past 7 hours. Denies vision or sound sensitivity. Patient points to $L$ temporal region as the location. Pt has tried taking acetaminophen to relieve the pain, but it did not work. Pt rates the pain as 10/10." This writing style focuses on the efficiency in recording plain data rather than communicating data analysis. Therefore, the scribe's note relies on the reader's interpretation being similar to the writer's, not taking into account that the reader may be analyzing the same data but reaching a different conclusion, or no conclusion at all. On the other hand, the benefit of the scribe's note lies in the reader's freedom to develop their own data analysis. With regards to objective data found in a scribe's note, only the pertinent data is recorded. However, if the reader is interested in data that the writer did not feel was 
pertinent, the reader will have to seek out the data elsewhere in the chart.

\section{The Storyteller}

In an effort to overcome the scribe's shortcomings and incorporate data analysis, the storyteller describes a story that logically flows into the ensuing plan. The storyteller may write, "Mr. Smith is a gentleman with a longstanding history of migraine headaches. Seven hours ago, he began to experience the worst headache of his life. It was unusual from his typical headaches; this headache was throbbing and stabbing, located in his left temporal region. Fortunately, there is no Brudzinski or Kernig's sign, which may be nonspecific. He attempted to relieve the pain with 3 concomitant acetaminophen tablets which proved to be ineffective. His family history is significant for aneurysms." With the storyteller's style, the reader is led to a logical conclusion based on the story. Unfortunately, it can appear that the differential, if not the final diagnosis, has been developed at the beginning, with the note likely leading to that final diagnosis rather than emphasizing differentials that have been excluded. Similar to the scribe, the storyteller may include only the pertinent objective data, but within a prose format. While this format may increase the readability of the note, it is more difficult to analyze compared to a table or list format.

\section{Conclusion}

The days of paper charting are numbered. The EHR revolution is underway and physicians are trying their best to transition as smoothly as possible. It is becoming clearer that EHR, with its many vaulted benefits, also allows for a plethora of typographic and stylistic mistakes; the writer now has to carefully proofread both the content and style of the note. Given these concerns, what is the ideal EHR note for clinician? One answer may be a note that combines the writing styles. For example, a scribe's interpretation of a patient's subjective story, coupled with a statistician's representation of the objective data, and finished with a storyteller's presentation of the assessment and plan. However, there are no published studies assessing the effect of clinical writing style on patient care. Further research is warranted to establish a clinical association.

Our challenge to you, the reader, is to apply the concepts presented here. The next day that you find yourself finishing an entry into an EHR, pause before finalizing your note, and reread your entry in its entirety. Do you have a style preference? If so, are there any small changes you can make that can help balance your style and improve communication with your readers? 


\section{Competing interests}

The authors declare that they have no competing interests.

\section{Authors' contributions}

All authors participated in the preparation of the manuscript, and read and approved the final manuscript.

\section{References}

1. Committee on Data Standards for Patient Safety, Board on Health Care Services. Key Capabilities of an Electronic Health Record System. Washington, DC: National Academy of Sciences; 2003.

2. Bernat JL. Ethical and quality pitfalls in electronic health records. Neurology. 201312;80(11):1057-61.

3. Hanlon JT. The electronic medical record: diving into a shallow pool? Cleve Clin J Med. 2010;77(7):408-11.

4. Josephson SA, Johnston SC, Hauser SL. Electronic medical records and the academic neurologist: when carrots turn into sticks. Ann Neurol. 2012;72(6):A5-6.

\section{Comment on this article:}
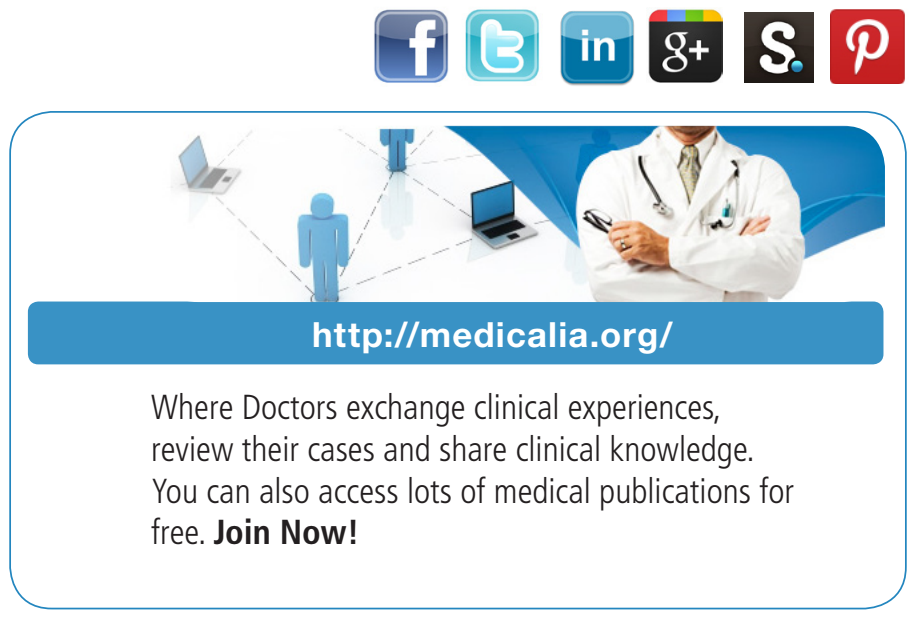

\section{Publish with iMedPub}

\section{http://www.imed.pub}

International Archives of Medicine is an open access journal publishing articles encompassing all aspects of medical science and clinical practice. IAM is considered a megajournal with independent sections on all areas of medicine. IAM is a really international journal with authors and board members from all around the world. The journal is widely indexed and classified Q1 in category Medicine. 